\title{
Examen médico forense del estado mental en el juzgado de guardia
}

\author{
Forensic mental status examination on duty court
}

\author{
JA. Guija Villa ${ }^{1,2}$ \\ L. Giner Jiménez ${ }^{2}$ \\ C. Romero de la Cruz ${ }^{1}$ \\ A. Guijarro Santoro ${ }^{1}$ \\ FJ. López Aguilar ${ }^{1}$ \\ ${ }^{1}$ Servicio de Psiquiatría \\ Forense. Instituto de \\ Medicina Legal de Sevilla. \\ ${ }^{2}$ Departamento de \\ Psiquiatría. \\ Universidad de Sevilla.
}

Correspondencia:

JA. Guija Villa

E-mail: julioaguija@gmail.com

Fecha de recepción:

08. DIC. 2013

Fecha de aceptación: 16. DIC. 2013

\section{Introducción}

La exploración psiquiátrica, o del estado mental, es la valoración transversal del comportamiento verbal y no verbal de una persona, incluyendo su nivel cognoscitivo y de vigilia ${ }^{1}$. Aplicado a la actividad médica forense en el juzgado de guardia, sería el estudio psicopatológico en el momento del reconocimiento, con el objeto de responder a las cuestiones planteadas por el juez con respecto al peritado, esto es, estado mental para declarar, relación del sujeto con sustancias adictivas y conveniencia de ingreso en una Unidad de Hospitalización Breve de Psiquiatría (UHB) (Tabla 1). Como puede observarse, no se considera la valoración de la imputabilidad en la guardia dada la imposibilidad de contar en ese momento con toda la información necesaria para establecer tan importante conclusión.

Las posibilidades en el juzgado de guardia son limitadas en cuanto a tiempo, espacio e información médica disponible. Por todo ello, hemos de ser conscientes de nuestras posibilidades y no ir más allá de lo que puede realizarse in situ con el peritado y de los objetivos que se plantean. En este sentido, la exploración del estado mental supone realizar un diagnóstico sindrómico (Tabla 2) y, con el fin de tomar medidas de carácter judicial, un pronóstico.

En psiquiatría, el diagnóstico se realiza de forma transversal y longitudinal. Así, transversalmente tratamos de poner de manifiesto el "estado del paciente aquí y ahora" mediante el estudio psicopatológico que nos revela posibles alteraciones de las funciones psíquicas en el momento del reconocimiento. Sin embargo, el estudio longitudinal, absolutamente necesario para establecer diagnósticos de acuerdo a las clasificaciones internacionales, requiere una serie de elementos con los cuales no se cuenta en el momento que nos ocupa:

- Derivados del propio sujeto: la realización de más de una entrevista en la que se pongan de manifiesto del modo más exhaustivo posible los antecedentes médicos, somáticos y psiquiátricos, así como antecedentes personales que ayuden a comprender a la persona.

- Documentales: en los que se encuentran registradas las posibles alteraciones físicas o psíquicas con expresión de la época de su vida en que han acontecido.

- Información de familiares que ayuden a concretar cuál ha sido la evolución de la persona.

La imposibilidad de obtener la información reseñada en el juzgado de guardia hace que el diagnóstico longitudinal, en la mayoría de las ocasiones, deba dejarse para la actividad forense posterior.

A diferencia de la entrevista clínica, en la guardia, la persona que es reconocida no siempre es un "paciente" en el sentido clásico médico. Hay que pensar que a veces ni el propio peritado conoce el motivo del reconocimiento, o bien sí lo conoce y espera obtener un beneficio de él. Por ello, hay que contar con posibles situaciones de simulación de síntomas o sobresimulación de éstos, sin que tal posibilidad sea el rector de nuestra actividad, ya que impediría apreciar con suficiente nitidez la realidad que se presenta.

\section{Objetivos del reconocimiento}

\section{En el detenido}

\section{Capacidad para declarar}

Deben evaluarse las funciones psíquicas (Figura 1) y sus posibles alteraciones. Así, se podrá determinar si en el momento en que presta declaración la persona tiene capacidad para conocer cuanto acontece a su alrededor, y si está libre de sintomatología psicótica que mediatice sus manifestaciones. Existen dos funciones que pueden influir en la capacidad para declarar, por distinto motivo: la consciencia y la psicomotricidad. 
El resto no impide la declaración, aunque sí hay que transmitir al juez y a las partes que intervienen en la declaración la situación psicopatológica del paciente y cómo puede influir. Respecto a la consciencia, si ésta no está alterada, el sujeto está capacitado para explicar las razones de "su conducta", aportando "su argumentación" (independientemente de la psicopatología que afecte en ese momento). En cuanto a la psicomotricidad, si se producen cuadros de agitación o de gran inquietud psicomotriz resulta físicamente inviable cualquier tipo de declaración.

Lo relevante es si la persona entiende qué se solicita de él y cuál es la trascendencia. Es requisito imprescindible que la consciencia no se encuentre alterada y que la psicomotricidad permita a la persona deponer con normalidad. Los trastornos de otras funciones psíquicas, como el pensamiento, la sensopercepción o la inteligencia, pueden alterar definitivamente la imputabilidad, pero no impedir el acto de la declaración; todo lo contrario, resultan un elemento de primer orden que ayuda a comprender al paciente en la realización del posterior informe sobre imputabilidad.

\section{Informe de adicción a sustancias tóxicas}

Como ya se ha mencionado, el diagnóstico de adicción requiere información longitudinal del paciente, de la cual casi siempre se carece en ese momento. Por ello, salvo que se disponga de acceso al historial médico acerca de la evolución clínica del detenido, en la mayoría de los casos lo más que puede hacerse es acreditar el estado físico y psíquico del paciente en el momento del reconocimiento, utilizando el término "según refiere" para todo aquello relacionado con el consumo de sustancias adictivas y que no podemos acreditar en nuestro reconocimiento.

\section{Ingreso en la UHB}

En los casos en que se detecta psicopatología activa que suponga un riesgo para el paciente o para terceros, nula consciencia de enfermedad, escaso apoyo social o familiar, y rechazo al tratamiento, el médico forense, tras el reconocimiento, puede y debe recomendar el ingreso en la UHB del hospital de referencia para valoración del caso y tratamiento si procede. Es decir, actúa como cualquier otro facultativo de acuerdo con el Art. 763 de la LEC.

\section{En la víctima}

Habitualmente, el reconocimiento que se efectúa en la guardia es de tipo físico. No obstante, en ocasiones hay que hacer referencia al estado psíquico. En estos

\section{Primarios}

Capacidad para declarar

Relación con sustancias adictivas

Ingreso en UHB de psiquiatría

\section{Secundarios}

Descripción psicopatológica

\section{Trastornos cognoscitivos}

2. Abuso de alcohol o drogas

3. Psicosis

4. Trastornos afectivos

5. Ansiedad o evitación irracionales

6. Quejas somáticas prominentes o exageradas

7. Conductas disociativas

8. Trastornos del sueño

9. Trastornos del control de los impulsos

10. Simulación, sobresimulación

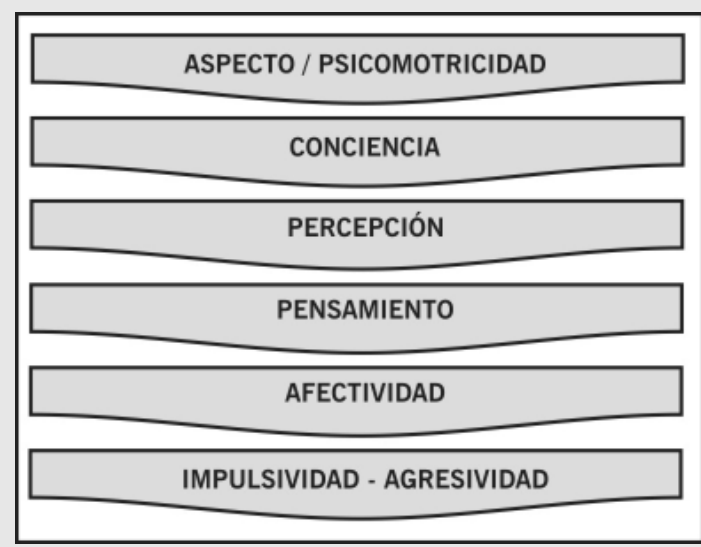

casos, al igual que en el detenido, lo relevante será el estudio psicopatológico que ponga de manifiesto el estado psíquico "aquí y ahora". La valoración acerca de posible secuelas es difícil realizarla en ese momento, en especial cuando la persona presenta algún tipo de vulnerabilidad (física o psíquica). Finalmente, hay que considerar la posibilidad de aparición de psicopatología diferida, lo que en psiquiatría no resulta extraño.
Tabla 1.

Objetivos del

reconocimiento forense en el juzgado de guardia.

Tabla 2.

Principales orientaciones diagnósticas (modificado de Bulbena).

Figura 1.

Algoritmo de exploración psicopatológica. 


\section{Métodología de la valoración psiquiátrica}

\section{Aspectos formales de la entrevista}

- Lugar: al igual que en cualquier reconocimiento médico, debe efectuarse en un despacho donde haya suficiente tranquilidad para permitir al médico forense explorar y al detenido expresarse.

- Tiempo: no existen tiempos medios establecidos, sino que dependerán de los objetivos de la entrevista. En general, debe emplearse el tiempo necesario para poder recabar la información, los signos y los síntomas necesarios que nos permitan elaborar una orientación diagnóstica.

- Actitud por parte del médico forense: la única diferencia entre el reconocimiento médico forense para determinar el estado mental y el reconocimiento médico clínico es su finalidad. Por ello, los principios de actuación deben estar regidos por los mismos principios éticos y deontológicos que cualquier actividad clínica no pericial.

- Seguridad y confidencialidad: la actividad pericial, como ya se ha señalado, debe estar regida por los mismos principios que la actividad médica clínica. Debe intentarse que el peritado se encuentre sin grilletes ni inmovilización alguna, y a ser posible sin la custodia policial presente. Estar liberado de grilletes permitirá apreciar actitudes, gestos, movimientos y cómo acompañan éstos a su estado de afectividad y al contenido de la expresión verbal. No obstante, cuando se aprecie riesgo de autoagresión o de agresión a otros, o de fuga, hay que tomar las medidas pertinentes, al igual que en psiquiatría clínica. Es deseable la confidencialidad en la entrevista, por lo que, en la medida de lo posible, la custodia policial no debe estar presente. Serán la pericia del médico y de las fuerzas de orden público la que determine el mejor modo de equilibrar seguridad y confidencialidad.

\section{Entrevista psicopatológica}

La exploración psicopatológica tiene como objetivo recabar signos y síntomas que nos indiquen la posible afectación de las funciones psíquicas ${ }^{2}$. Es conveniente llevar una sistemática con idea de realizarla de forma completa. Teniendo en consideración la especial situación que nos ocupa, y con los objetivos antes señalados, en la entrevista psicopatológica es imprescindible valorar la psicomotricidad, la consciencia, la percepción, el pensamiento, la afectividad y la impulsividad-agresividad ${ }^{3}$ (Figura 1 ). Previamente a la entrevista propiamente dicha, deben examinarse los signos y síntomas que nos hagan sospechar el consumo de tóxicos ${ }^{4}$ (Tabla 3).

- Psicomotricidad: es evaluable desde el momento en que se ve al sujeto. De esta forma podremos observar si los movimientos corporales están disminuidos o aumentados, pudiendo llegar incluso a la agitación psicomotriz, que sería motivo de suspensión del reconcocimiento y de derivación a la UHB.

- Consciencia: la exploración se realizará de mayor a menor gravedad de afectación de las funciones propias de ésta, comenzando por la orientación temporo-espacial y en persona, y si esta está conservada se podrá pasar a la atención, el razonamiento, el cálculo y la abstracción ${ }^{5}$. La valoración conjunta de consciencia, atención y memoria puede basarse en la aplicación del Mini-Mental test ${ }^{6}$. Una vez descartado que haya afectación de la consciencia se procederá a la valoración de la sintomatología psicótica activa a través de la esfera perceptiva y de pensamiento.

- Percepción: detectar la presencia de alteraciones y, en su caso, realizar una descripción fina de ellas con referencia al campo perceptivo ${ }^{7}$.

- Pensamiento, abordado desde tres puntos de vista: propiedad, curso y contenido ${ }^{8}$. Propiedad: indagar sobre la intimidad del pensamiento, preguntando sobre si algo o alguien interfiere en él. Curso: observar cómo se desarrolla la entrevista e identificar si es rápido o lento, si se detiene en multitud de detalles o pierde el hilo cuando habla. Por último, pero no menos importante, el contenido del pensamiento mediante la búsqueda de ideación delirante. La exploración de la ideación delirante puede efectuarse de manera indirecta preguntando por las relaciones sociales en su entorno (trabajo, familia o vivienda). En caso de no detectarla, preguntar directamente sobre los contenidos delirantes más frecuentes, como son los de perjuicio o de referencia. En caso de que sea también negativo tendremos otra oportunidad de abordaje del contenido del pensamiento cuando se valore la afectividad.

- Afectividad: preguntar directamente sobre su estado de ánimo. En caso de afectación del humor, la respuesta nos permitirá profundizar en las causas que el sujeto esgrime para tal situación; de este modo, un estado de ánimo ansioso o temeroso en relación a sentirse observado o en peligro nos orienta de nuevo a la ideación delirante. Otras indicaciones de estado de ánimo triste, o incluso eufórico, también permiten ahondar en las causas o en las consecuencias del estado 


\begin{tabular}{ll}
\hline Alcohol & Resto de sustancias adictivas \\
\hline Eritema palmar & Midriasis \\
Telangiectasias & Miosis \\
Sudoración & Inyección conjuntival \\
Temblor distal & Lagrimeo \\
Ansiedad & Nistagmo \\
Rinofima & Rinorrea \\
Anillo corneal senil & Hipertensión/hipotensión \\
Debilidad & Taquicardia \\
Impotencia & Hipertermia/hipotermia \\
Delgadez/anorexia & Bradipnea \\
Hepatomegalia & Úlceras \\
Dolores abdominales & Signos de venopunción \\
Convulsiones & Temblor distal \\
& Hiperreflexia/hiporreflexia \\
& Convulsiones \\
& Estreñimiento \\
\hline
\end{tabular}

Tabla 3.

Signos y síntomas relacionados con el consumo de sustancias. afectivo en la vida diaria o en el motivo de su valoración pericial. Complementando la esfera afectiva deben valorarse los aspectos instintivos relacionados con ella, como son el sueño, la alimentación y la sexualidad. El último apartado no debe prolongarse en la entrevista más allá de lo puramente necesario para ver si están afectados o no.

- Impulsividad-agresividad: mediante preguntas que impliquen una decripción de sí mismo acerca de su impulsividad, irritabilidad, agresividad y valoración de tiempo de respuesta ante situaciones estresantes o frustrantes, así como de las posibles consecuencias de sus actos. Es conveniente recoger conductas autoagresivas, autolesivas y suicidas.

- Otros aspectos: la inteligencia grosso modo según la trayectoria vital y ayudándose de alguna pregunta sobre diferencias y similitudes. Finalmente, la personalidad no va a aportar mucho en la valoración en ese momento, y consumiría mucho tiempo, por lo que será una exploración accesoria pero no necesaria.

\section{Elaboración del informe}

Por sus objetivos, el informe debe ser concreto y responder a las necesidades judiciales, y posteriormente periciales. Así, debe concretarse en:

- Filiación.

- Antecedentes médicos y personales de interés en la causa. No debe hacerse un barrido de enfermedades médicas sin relación alguna con el hecho o de nulo interés para comprender al peritado.

- Estado psicopatológico actual.

- Consideraciones médico-forenses acerca de los posibles motivos del reconocimiento: capacidad para declarar, consumo de sustancias adictivas, ingreso en UHB.

- Conclusiones.

Los autores declaran no tener conflictos de intereses. 


\section{Bibliografía}

1. Bulbena A. Exploración psiquiátrica. En: Vallejo Ruiloba J, editor. Introducción a la psicopatología y a la psiquiatría. $6^{a}$ ed. Barcelona: Elsevier España S.L.; 2006. p. 119-28.

2. Sims A. Síntomas mentales: introducción a la psicopatología descriptiva. Madrid: Ed. Triacastela; 2008.

3. García-Moreno M, Poza B. Entrevista psiquiátrica en urgencias. En: Giner L, Carballo JJ, García-Thuring L, Baca-García E, editores. Urgencias en psiquiatría. Guía práctica para residentes. Madrid: Enar; 2006. p. 1-8.

4. Jiménez Treviño L, Correas lauffer J. El paciente drogodependiente. En: Chinchilla A, editor. Breviario de urgencias psiquiátricas. Barcelona: Elsevier España S.L.; 2011. p. 81-91.

5. Muñoz P, Anguiano JB, Mondragón MS. Conciencia. En: Eguiluz Uruchurtu JI, editor. Introducción a la psicopatología. Madrid: IM\&C 2001; p. 37-64.

6. Folstein MF, Folstein SE, Mcpugh PR. Mini-mental state. A practical method for granding the cognitive state of patients for the clinician. J Psychiatr Res. 1975;12:189-98.

7. Hamilton H. Trastornos de la percepción. Psicopatología clínica de Fish. Madrid: Emalsa; 1996. p. 19-42.

8. Serrallonga J. Trastornos del contenido del pensamiento. En: Vallejo J, editor. Árboles de decisión en psiquiatría. Barcelona: Jims; 1999. p. 14-5. 